\title{
Relative frequency, clinical, neuroimaging, and postsurgical features of pediatric temporal lobe epilepsy
}

L.V. Sales ${ }^{1}$, T.R. Velasco ${ }^{1}$ S. Funayama ${ }^{1}$, L.T. Ribeiro ${ }^{1}$, L.P. Andrade-Valença ${ }^{1}$, L. Neder ${ }^{3}$, R.M.F. Fernandes ${ }^{1}$, D. Araujo Jr. ${ }^{2}$, H.R. Machado ${ }^{4}$, A.C. Santos ${ }^{2}$ and J.P. Leite ${ }^{1}$
${ }^{1}$ Departamento de Neurologia, ${ }^{2}$ Departamento de Clínica Médica, ${ }^{3}$ Departamento de Patologia, ${ }^{4}$ Departamento de Cirurgia,

Faculdade de Medicina de Ribeirão Preto, Universidade de São Paulo, Ribeirão Preto, SP, Brasil
Correspondence

J.P. Leite

Departamento de Neurologia

FMRP, USP

Av. Bandeirantes, 3900

14049-900 Ribeirão Preto, SP

Brasil

Fax: +55-16-3633-0760

E mail: jpleite@fmrp.usp.br

Research supported by FAPESP (Nos. 99/11729-2 and 00/01773-3 to J.P. Leite and L.V. Sales, respectively), CNPq, PRONEX and FAEPA.

Received December 20, 2005

Accepted June 20, 2006

\begin{abstract}
We describe the relative frequency, clinical features, neuroimaging and pathological results, and outcome after pharmacological or surgical intervention for a series of pediatric patients with temporal lobe epilepsy (TLE) from an epilepsy center in Brazil. The medical records of children younger than 12 years with features strongly suggestive of TLE were reviewed from January 1999 to June 1999. Selected children were evaluated regarding clinical, EEG, and magnetic resonance imaging (MRI) investigation and divided into three groups according to MRI: group $1(\mathrm{G} 1, \mathrm{~N}=9)$, patients with hippocampal atrophy; group $2(\mathrm{G} 2, \mathrm{~N}=10)$, patients with normal MRI, and group 3 (G3, N $=12$ ), patients with other specific temporal lesions. A review of 1732 records of children with epilepsy revealed 31 cases with TLE (relative frequency of $1.79 \%$ ). However, when the investigation was narrowed to cases with intractable seizures that needed video-EEG monitoring $(\mathrm{N}=68)$ or epilepsy surgery $(\mathrm{N}=32)$, the relative frequency of TLE increased to $19.11(13 / 68)$ and $31.25 \%$ (10/32), respectively. At the beginning of the study, 25 of 31 patients had a high seizure frequency $(80.6 \%)$, which declined to 11 of $31(35.5 \%)$ at the conclusion of the study, as a consequence of pharmacological and/or surgical therapy. This improvement in seizure control was significant in $\mathrm{G} 1(\mathrm{P}<0.05)$ and G3 $(\mathrm{P}<0.01)$ mainly due to good postsurgical outcome, and was not significant in G2 ( $P>0.1$, McNemar's test). These results indicate that the relative frequency of TLE in children was low, but increased considerably among cases with pharmacoresistant seizures. Patients with specific lesions were likely to undergo surgery, with good postoperative outcomes.
\end{abstract}

Key words

- Temporal lobe epilepsy

- Children

- Etiology

- Prognosis

- Developing countries 


\section{Introduction}

Temporal lobe epilepsy (TLE) is the most common epileptic syndrome in adolescents and adults, and hippocampal sclerosis (HS) its most frequent pathological substrate (1). Studies of pediatric series have shown that there are substantial differences compared to adult series. First, the clinical features of temporal lobe seizures tend to be simpler in younger children and ictal and interictal EEG findings are less localized or falsely lateralized (2). Second, the relative frequency of TLE is lower in children than in adults as also is the frequency of HS as the pathological substrate in surgical series $(3,4)$. Finally, in developed countries, community-based cohort studies of pediatric new-onset TLE have shown that neuroimaging features are within normal limits in the majority of cases, which contrasts to the high frequency of hippocampal atrophy and T2 signal changes in adult series $(5,6)$.

The relative frequency and etiologic profile of TLE in children have been poorly described in developing countries. This information is particularly relevant in view of the potential etiologies commonly associated with low-socioeconomic status $(7,8)$. In recent years, some developing countries have witnessed a substantial increase in the number of patients referred to centers with a multidisciplinary structure, a fact that has permitted a systematic evaluation of patients with epilepsy $(9,10)$.

In the present study, we describe the relative frequency, clinical features, neuroimaging, and pathological results of a series of pediatric patients with TLE from a mustidisciplinary epilepsy center in Brazil. Our results indicated that the relative frequency of TLE in children was low, but was considerably higher among cases with pharmacoresistant seizures. In addition, we found that the etiologic profile of the disease was similar to that reported in developed countries.

\section{Patients and Methods}

Patients were evaluated at the outpatient clinic and video-EEG unit of the University Hospital, Ribeirão Preto School of Medicine, University of São Paulo, Ribeirão Preto, SP, Brazil, between January 1999 and December 2001, using standardized protocols previously published. Parents or caregivers of the children signed a free and informed consent and the protocol was approved by the Ethics Committee of the Institution (HCRP \#7366/99) (11).

The children were selected by retrospective evaluation of the medical records of epileptic patients younger than 12 years with features strongly suggestive of TLE as proposed by the classification of the International League Against Epilepsy (ILAE) (12). These features were: 1) two or more complex partial seizures often beginning with motor arrest followed by automatisms, with a duration $>1 \mathrm{~min}$, and gradual recovering; 2) simple partial seizures characterized by autonomic or psychic symptoms and sensory phenomena such as olfactory and auditory ones, most commonly epigastric rising sensation.

We excluded patients with clinical and laboratory features that placed the diagnosis of TLE in doubt, including focal abnormalities on neurological examination, generalized or extratemporal EEG spikes, extratemporal lesions on magnetic resonance imaging (MRI), severe mental retardation, and genetic syndromes. The selected records were reviewed in full detail regarding a family history of epilepsy, age of first seizure, and presence of an initial precipitating injury (IPI). As a possible IPI we considered a previous history of meningitis, complex febrile seizures (prolonged, focal or multiple), trauma with loss of conscience, perinatal hypoxemic insult, and status epilepticus.

All patients were prospectively followed up for 3 years (1999-2001). During the follow-up period, the patients were evaluated 
regarding seizure frequency and divided into groups as having low or high seizure frequency. Low seizure frequency was indicated when patients had one seizure every three months or less for more than two years. High seizure frequency was indicated when patients had one seizure every month or more. Children with low seizure frequency were seen at least once a year. Children with a high number of seizures (more than 1 per month) were seen monthly.

\section{Neuroimaging acquisition and analysis}

MRI was performed with a 1.5 Tesla system Magneton Vision apparatus ( $\mathrm{Si}$ emens, Erlangen, Germany). The protocol included: i) a sagittal T1-weighted 3-D gradient echo sequence covering the whole head $\left(\mathrm{TR}=9.7 \mathrm{~ms} ; \mathrm{TE}=4 \mathrm{~ms}\right.$; flip angle $\left.=12^{\circ}\right)$, with a field-of-view (FOV) of $256 \mathrm{~mm}$, a $256 \times 256$ matrix, and a partition thickness of $1 \mathrm{~mm}$, allowing an isometric voxel of 1 $\mathrm{mm}$; ii) a coronal turbo fluid attenuation inversion recovery sequence $(\mathrm{TR}=9000$ $\mathrm{ms}$; TI $=2200 \mathrm{~ms}$; TE $=119 \mathrm{~ms}$ ), perpendicular to the hippocampal axis, sixteen 5$\mathrm{mm}$ thick slices with a $0.5-\mathrm{mm}$ interslice gap, a 192 × 256 matrix, and FOV of 240 $\mathrm{mm}$; iii) an axial turbo spin-echo T2-weighted sequence $(\mathrm{TR}=5400 \mathrm{~ms} ; \mathrm{TE}=99 \mathrm{~ms})$ parallel to the corpus callosum, twenty 5$\mathrm{mm}$ thick slices with a 1-mm interslice gap, a 192 x 256 matrix, and FOV of $240 \mathrm{~mm}$, and iv) a coronal $\mathrm{T} 2$-weighted turbo spin-echo $(\mathrm{TR}=5400 \mathrm{~ms}$; TE $=99 \mathrm{~ms})$, FOV of 200 $\mathrm{mm}$, a 512 x 512 matrix, and twenty 3-mm thick slices with no interslice gap.

The volumetric 3-D gradient echo sequences were reformatted using Multiplanar Reconstruction Program (Siemens) on the coronal plane, perpendicular to the largest axis of the hippocampus. Twenty contiguous 2-mm thick slices were chosen, including the entire hippocampal volume and were used for manual segmentation and volumetric analysis. Images were transferred to an
IBM-PC workstation (Windows 2000) and processed for volumetry using specific software (Image J - NIH software). The absolute and relative values of the hippocampus were compared to a control group. Hippocampal atrophy was defined as a volume smaller than two standard deviations of the average for the control group $(\mathrm{N}=9)$ analyzed by the same method. For the control group, nine normal images from age-matched patients without epilepsy were analyzed.

Patients were divided into three groups according to neuroimaging features (MRI), as follows: group $1(\mathrm{G} 1, \mathrm{~N}=9)$ - patients with hippocampal atrophy and/or increased signal in the T2-weighted sequences; group $2(\mathrm{G} 2, \mathrm{~N}=10)$ - patients with normal MRI, and group $3(\mathrm{G} 3, \mathrm{~N}=12)$ - patients with other specific temporal lesions (tumors and malformations of cortical development). Six patients were additionally submitted to $\mathrm{CT}$ scans.

\section{EEG and video-EEG monitoring}

EEGs were obtained with a standard 21channel EEG apparatus (Nihon Kohden model Neurofax, Tokyo, Japan). Electrodes were placed according to the international 10-20 system. Data were reviewed by experienced neurophysiologists. Cases with intractable seizures and tumor cases were submitted to video-EEG monitoring. When preoperative data were localized to the temporal lobe, patients were submitted to a standard anterior and mesial temporal 'en bloc' resection including 2-3 cm of the hippocampus. The final etiologic diagnosis was confirmed by histopathological examination.

\section{Statistical analysis}

We applied the Kolmogorov-Smirnov test to define the distribution of the variables. When variables presented a normal distribution we used parametric tests such as the Student $t$-test or analysis of variance 
(ANOVA). When variables did not present a normal distribution we used the non-parametric Mann-Whitney or Kruskal-Wallis tests. For categorical variables we applied the exact Fisher test for independent observations and the McNemar test for paired observations.

\section{Results}

The demographic and clinical data of the patients are presented in Table 1. Of the 1732 medical records of children with epilepsy reviewed during the study period, 31 fulfilled the inclusion criteria for pediatric TLE, corresponding to a relative frequency of $1.79 \%$. During the same period, $19.11 \%$ (13 of 68) patients with intractable seizures evaluated in the pediatric video-EEG monitoring unit had neuroimaging and neurophysiological evidence of TLE. Of the 13 patients who had video-EEG investigation, 10 were submitted to temporal lobectomy, accounting for a relative frequency of $31.25 \%$ (10 of 32) of the total pediatric surgical procedures.

The mean age was 7.87 years, with a range of 3 to 12 years. The mean age at seizure onset was 3.84 years (range: 2 months to 11 years). There was a tendency to an inverse relationship between age at seizure onset and seizure frequency (Mann-Whit- ney test, $\mathrm{P}=0.06)$. Of the 31 children, 20 $(64.5 \%)$ had a positive family history of epilepsy. A previous history of IPI was present in 21 children (67.7\%), and the events were the following: complex febrile seizures $(\mathrm{N}=9)$, status epilepticus $(\mathrm{N}=8)$, meningitis $(\mathrm{N}=3)$, and perinatal hypoxia $(\mathrm{N}=1)$. Eight of 9 G1 patients (88.88\%) and 9/10 G2 patients $(90 \%)$ had an IPI. This proportion was significantly greater than the 4/12 (33.3\%) found in G3 (Fisher exact test, G1 vs $\mathrm{G} 3 ; \mathrm{P}=0.024 ; \mathrm{G} 2$ vs $\mathrm{G} 3, \mathrm{P}=0.011)$. Developmental disabilities were seen in 13 of 31 patients (43.7\%).

Focal seizures were observed in $100 \%$ of cases, and secondary generalized seizures in 7 of $31(22.6 \%)$. At the beginning of the study (1999), $80.6 \%$ of patients had a high frequency of seizures, with a decline to $35.5 \%$ at the conclusion of the study (2001) as a consequence of pharmacological (21 patients) or surgical (10 patients) therapy (see Table 2). Regarding specific groups, in G1 $(\mathrm{N}=9), 2$ of 2 patients became seizure free due to surgical therapy, and 4 of 6 patients improved due to pharmacotherapy (3 seizure free and 1 with one seizure in 4 months). In $\mathrm{G} 2(\mathrm{~N}=10), 4$ of 10 patients had an improvement in seizure control due to pharmacotherapy ( 3 seizure free and 1 with one seizure in 4 months). Finally, in $\mathrm{G} 3(\mathrm{~N}=12)$, 8 of 8 patients became seizure free due to

Table 1. Demographic and clinical features of children with temporal lobe epilepsy (TLE).

$\begin{array}{ll}\text { TLE pediatric patients/all pediatric epileptic patients } & 31 / 1732(1.79 \%) \\ \text { Video-EEG TLE patients/video-EEG intractable epileptic patients } & 13 / 68(19.11 \%) \\ \text { Temporal lobectomy/all pediatric epilepsy surgery patients } & 10 / 32(31.25 \%) \\ \text { Sex } & 18 \text { females, } 13 \text { males } \\ \text { Age at enrollment } & 7.87 \pm 2.78(3-12 \text { years) } \\ \text { Age at seizure onset } & 3.84 \pm 3.24(2 \text { months-11 years) } \\ \text { Epilepsy duration } & 4.34 \pm 2.66(2 \text { months-10 years) } \\ \text { Family history of epilepsy } & 20 / 31(64.5 \%) \\ \text { Presence of initial precipitating injury } & 21 / 31(67.7 \%) \\ \text { Developmental problems } & 13 / 31(43.7 \%)\end{array}$

The 1732 patients were treated in the outpatient clinic of a tertiary level University hospital from 1999 to 2001. Data for age at enrollment, age at seizure onset and epilepsy duration are reported as means \pm SD and range for 31 TLE pediatric patients. 
surgical therapy, and 2 of 4 patients improved due to pharmacotherapy and are seizure free. Overall, the improvement in seizure control was significant in $\mathrm{G} 1(\mathrm{P}<0.05)$ and in $\mathrm{G} 3(\mathrm{P}<0.01)$, and was not significant in $\mathrm{G} 2$ ( $\mathrm{P}>0.1$, McNemar's test for paired observations). No differences were found among groups regarding age at enrollment, age at seizure onset, epilepsy duration, or family history of epilepsy.

Thirteen children had a normal EEG, 3 had bilateral temporal interictal spikes, and 16 had unilateral interictal spikes ( 15 temporal, 1 temporal-occipital). In $94 \%$ of cases $(15 / 16)$ unilateral spikes were ipsilateral to the MRI findings. Thirteen children were submitted to video-EEG monitoring, with 12 showing complex partial seizures with motor automatisms and one tonic asymmetric seizures. Ictal EEG onset was temporal in 7 cases, hemispheric in 2 and diffuse in 2 cases. In the patient with tonic asymmetric seizures, the interictal and ictal EEG onset was localized in the temporal lobe.

Regarding the neuroimaging features, we found 9 patients with features suggestive of HS (G1), 10 patients with no MRI abnormality (G2), and 12 patients with specific etiologies (G3; Table 2). The hippocampal volumes of $6 \mathrm{G} 1$ patients were smaller than 2 standard deviations of the average control group, and the remaining three cases had a diagnosis of probable HS based on visual analysis. In G2 and G3, hippocampal volumes were within normal limits. Ten patients, 2 from G1 and 8 from G3, were submitted to temporal lobectomy. Histopathological examination confirmed the diagnosis of HS in the two G1 surgical cases. The specific etiologies in G3 were cortical dysplasia $(\mathrm{N}=6)$, dysembryoplastic neuroepithelial tumor $(\mathrm{N}=1)$, and tanycytic ependymoma $(\mathrm{N}=1)$.

Finally, MRI or CT scans showed no lesions supporting a diagnosis of neurocysticercosis, tuberculosis, echinococcosis (hydatidosis, or hydatid disease), or any other etiology commonly associated with developing countries (13-17).

\section{Discussion}

The present study, conducted at a University hospital which provided diagnostic evaluation and treatment to children with epilepsy whose seizures ranged from easily controlled (primary care) to intractable (tertiary care), indicated that the relative frequency of TLE pediatric epilepsy patients was low, 31/1742 (1.8\%). However, when

Table 2. Clinical and magnetic resonance imaging (MRI) findings of children with temporal lobe epilepsy according to group.

\begin{tabular}{llll}
\hline $\begin{array}{l}\text { Etiology } \\
\text { MRI findings }\end{array}$ & $\begin{array}{l}\text { Group 1 }(\mathrm{N}=9) \\
\mathrm{HA}(\mathrm{N}=8) \text { or } \\
\text { T2 hypersignal }(\mathrm{N}=1)\end{array}$ & $\begin{array}{l}\text { Group 2 }(\mathrm{N}=10) \\
\text { Normal MRI }\end{array}$ & $\begin{array}{l}\text { Group 3 }(\mathrm{N}=12) \\
\text { Specific etiology } \\
\text { tumors }(\mathrm{N}=2) \text {, arachnoid cyst }(\mathrm{N}=1), \\
\text { choroid cyst }(\mathrm{N}=1), \text { dysplasia }(\mathrm{N}=8)\end{array}$ \\
\hline Hippocampal volumetry & 6 patients with HA & Normal & Normal \\
Initial precipitating injury & 8 present, 1 absent & 9 present, 1 absent & 4 present, 8 absent \\
HSF in 1999 $[25 / 31(80.6 \%)]$ & $7 / 9$ patients $(77.7 \%)^{*}$ & $8 / 10$ patients $(80 \%)^{+}$ & $10 / 12$ patients $(83.3 \%)^{* *}$ \\
HSF in 2001 $[11 / 31(35.5 \%)]$ & $3 / 9$ patients $(33.3 \%)^{*}$ & $6 / 10$ patients $(60 \%)^{+}$ & $2 / 12$ patients $(16.6 \%)^{* *}$ \\
Age at enrollment & 8.67 years & 8 years & 7.17 years \\
Age at seizure onset & 3.43 years & 2.0 years & 3.28 years \\
Epilepsy duration & 5.74 years & 4.38 years & 3.13 years \\
Family history & 6 yes, 3 no & 7 yes, 3 no & 7 yes, 5 no
\end{tabular}

$\mathrm{HA}=$ hippocampal atrophy; $\mathrm{HSF}=$ high seizure frequency.

G1 vs G3 (Fisher exact test; $\mathrm{P}=0.024 ; \mathrm{OD}=16 ; 95 \% \mathrm{Cl}=1.450$ to 176.56 ). $\mathrm{G} 2$ vs $\mathrm{G} 3$ (Fisher exact test; $\mathrm{P}=0.011 ; \mathrm{OD}=18 ; 95 \% \mathrm{Cl}=1.649$ to 196.42). McNemar's test for paired observations 1999 vs 2001: ${ }^{*} P<0.05,{ }^{\star \star} P<0.01,{ }^{+} P>0.10$. 
the investigation was narrowed to cases with intractable seizures that needed video-EEG monitoring $(\mathrm{N}=68)$ or epilepsy surgery $(\mathrm{N}$ $=32$ ), the relative frequency of TLE increased to 19.11 (13 of 68) and $31.25 \%$ (10 of 32), respectively. The overall low relative frequency is in agreement with data from retrospective studies on children with TLE. Murakami et al. (3), in a retrospective study of 2319 epileptic patients with childhood onset of the disease, found a relative frequency of limbic epilepsy in $19(0.82 \%)$. Series from pediatric epilepsy surgery centers have pointed out that TLE represents a larger proportion of intractable cases and that malformations of cortical development and tumors in the temporal lobe are much more common than mesial temporal lobe epilepsy in children under 12 years of age $(2,18)$.

The low relative frequency of TLE in children should explain the relatively small number of patients in the present series and in those reported elsewhere (2,18-20).

It is important to emphasize that the present study was hospital-based and not a population-based study. There may be children with TLE whose seizures are easily controlled with medication or so discrete that they never come to the attention of an epileptologist. Other methodological aspects of our study that should be considered are: 1) due to the retrospective nature of the present pediatric study, some information might have been lost, despite the relatively short period between the onset of epilepsy and the medical interview. However, most of the information could be retrieved during prospective follow-up; 2) determining whether a seizure is of temporal lobe origin in children can be difficult. This is particularly relevant in children younger than 6 years, whose seizures can present a broad spectrum of semiologic characteristics and in those with a normal MRI (21). However, we used restrictive ILAE criteria for TLE, and cases with mental retardation and extratemporal spikes were excluded. In fact, this may have biased our sample to cases with a more localized spectrum of TLE. For example, it is well known that patients with West syndrome, although classified as having generalized epilepsy, may have structural lesions restricted to the temporal lobe (22); 3) since one of the features of the natural history of mesial TLE is the onset of complex partial seizures at the end of the first decade or during the teen years, the upper limit of 12 years of age for our series may have excluded some mesial TLE patients whose seizure onset might have occurred after this period.

Despite these possible limitations, we believe that our findings provide important comparative information on the relative frequency of pediatric TLE with a different spectrum of seizure control (from low to high frequency of seizures).

In the present study, we observed a tendency to an inverse relationship between age at seizure onset and seizure frequency at the beginning of the study (Mann-Whitney test, $\mathrm{P}=0.06$ ), which is somewhat in agreement with other studies reporting that early seizure onset is significantly associated with poor seizure control $(23,24)$.

We detected a high frequency of IPI, particularly in children with evidence of hippocampal atrophy (G1) or normal MRI (G2), which was significantly different from children with specific MRI lesions (Fisher exact test, $\mathrm{P}=0.011$ ). Although pediatric cohort studies have shown a frequency of IPI in $29 \%$ of TLE cases (5), retrospective adult studies have indicated a wide variability of IPI, ranging from 30 to over $80 \%$ (25-27). This variability may reflect the use of different criteria for defining IPI, the relative contribution of typical mesial TLE in those series, and the interval between IPI and medical interview.

One surprising finding of our study was the high frequency of IPI in children with normal MRI (G2, 9 of 10). One may ques- 
tion if some of these children would develop mesial TLE in the future as a consequence of repeated IPI or chronic seizures (28). Although it is well known that prolonged febrile convulsions can produce acute MRI signal changes in the hippocampus and subsequent development of hippocampal atrophy (29), the contribution of brief seizures to hippocampal sclerosis is controversial. Several studies support the view that hippocampal sclerosis is likely to be an acquired pathology, and most of the neuronal loss occurs as a consequence of the precipitating injury $(25,26,30,31)$. However, there is recent evidence that HS may be acquired after brief generalized tonic-clonic seizures in adults (32). Prospective volumetric studies of our children with normal MRI will be necessary to document the progression to hippocampal atrophy if it occurs.

The results of the present study support the importance of MRI findings in epilepsy surgery, provided that patients with specific lesions are likely to undergo surgery, with good postoperative outcomes $(2,18)$. On the other hand, surgical treatment for children with normal MRI represents one of the most challenging issues in pediatric epilepsy surgery (33). This is particularly important due to the fact that surgical treatment has the potential to provide freedom from disabling seizures as opposed to continuing pharmacotherapy, reducing the risk of long-term morbidity and mortality (34).

Finally, we found no lesions on the MRI or CT scans supporting the diagnosis of etiologies commonly associated with epilepsy in developing countries (13-17). The following considerations may explain this finding. First, neurotuberculosis and echinococcosis are not endemic in Brazil. Second, although neurocysticercosis is very prevalent in Brazil, the likelihood of children getting infected is smaller than for adolescents and adults (35). In addition, there is evidence that neurocysticercosis might be a coincidental pathology in TLE which does not play a role in epileptogenesis (7). Finally, several etiologies associated with poor primary health care in developing countries may be causal factors of more diffuse brain involvement rather than involvement limited to the temporal lobe $(36,37)$.

\section{Acknowledgments}

The authors thank the staff of the Epilepsy Outpatient Clinics and Video-EEG Monitoring Unit. We also thank Geraldo Cássio dos Reis for statistical advice.

\section{References}

1. Engel J Jr. Mesial temporal lobe epilepsy: what have we learned? Neuroscientist 2001; 7: 340-352.

2. Wyllie E, Chee M, Granstrom ML, DelGiudice E, Estes M, Comair Y, et al. Temporal lobe epilepsy in early childhood. Epilepsia 1993; 34: 859-868.

3. Murakami N, Ohno S, Oka E, Tanaka A. Mesial temporal lobe epilepsy in childhood. Epilepsia 1996; 37 (Suppl 3): 52-56.

4. Bocti C, Robitaille Y, Diadori P, Lortie A, Mercier C, Bouthillier A, et al. The pathological basis of temporal lobe epilepsy in childhood. Neurology 2003; 60: 191-195.

5. Harvey AS, Berkovic SF, Wrennall JA, Hopkins IJ. Temporal lobe epilepsy in childhood: clinical, EEG, and neuroimaging findings and syndrome classification in a cohort with new-onset seizures. Neurology 1997; 49: 960-968.

6. Williamson PD, French JA, Thadani VM, Kim JH, Novelly RA, Spen- cer SS, et al. Characteristics of medial temporal lobe epilepsy: II. Interictal and ictal scalp electroencephalography, neuropsychological testing, neuroimaging, surgical results, and pathology. Ann Neurol 1993; 34: 781-787.

7. Leite JP, Terra-Bustamante VC, Fernandes RM, Santos AC, Chimelli $\mathrm{L}$, Sakamoto $\mathrm{AC}$, et al. Calcified neurocysticercotic lesions and postsurgery seizure control in temporal lobe epilepsy. Neurology 2000; 55: 1485-1491.

8. Garcia HH, Gonzalez AE, Evans CA, Gilman RH. Taenia solium cysticercosis. Lancet 2003; 362: 547-556.

9. Mani KS, Rangan G. Delivery of health care and socioeconomic issues: India. In: Engel J Jr, Pedley TA (Editors), Epilepsy: A comprehensive textbook. Philadelphia: Lippincott-Raven; 1997. p 28352840.

10. Guerreiro CAM. Delivery of health care and socioeconomic issues: 
Brazil. In: Engel J Jr, Pedley TA (Editors), Epilepsy: A comprehensive textbook. Philadelphia: Lippincott-Raven; 1997. p 2817-2823.

11. Velasco TR, Wichert-Ana L, Leite JP, Araujo D, Terra-Bustamante VC, Alexandre V Jr, et al. Accuracy of ictal SPECT in mesial temporal lobe epilepsy with bilateral interictal spikes. Neurology 2002; 59: 266-271.

12. Proposal for revised classification of epilepsies and epileptic syndromes. Commission on Classification and Terminology of the International League Against Epilepsy. Epilepsia 1989; 30: 389-399.

13. Andronikou S, Welman CJ, Kader E. Classic and unusual appearances of hydatid disease in children. Pediatr Radiol 2002; 32: 817828.

14. Carpio A, Escobar A, Hauser WA. Cysticercosis and epilepsy: a critical review. Epilepsia 1998; 39: 1025-1040.

15. Khaldi M, Mohamed S, Kallel J, Khouja N. Brain hydatidosis: report on 117 cases. Childs Nerv Syst 2000; 16: 765-769.

16. Salgado $\mathrm{P}$, Del Brutto $\mathrm{OH}$, Talamas $\mathrm{O}$, Zenteno MA, RodriguezCarbajal J. Intracranial tuberculoma: MR imaging. Neuroradiology 1989; 31: 299-302.

17. Tuzun M, Altinors N, Arda IS, Hekimoglu B. Cerebral hydatid disease CT and MR findings. Clin Imaging 2002; 26: 353-357.

18. Duchowny M, Levin B, Jayakar P, Resnick T, Alvarez L, Morrison G, et al. Temporal lobectomy in early childhood. Epilepsia 1992; 33: 298-303.

19. Murakami T, Mita S, Tokunaga M, Maeda H, Ueyama $\mathrm{H}$, Kumamoto $\mathrm{T}$, et al. Hereditary cerebellar ataxia with Leber's hereditary optic neuropathy mitochondrial DNA 11778 mutation. J Neurol Sci 1996; 142: 111-113.

20. Mohamed A, Wyllie E, Ruggieri P, Kotagal P, Babb T, Hilbig A, et al. Temporal lobe epilepsy due to hippocampal sclerosis in pediatric candidates for epilepsy surgery. Neurology 2001; 56: 1643-1649.

21. Holmes GL. Partial seizures in children. Pediatrics 1986; 77: 725731.

22. Hamano S, Tanaka M, Kawasaki S, Nara T, Horita H, Eto $\mathrm{Y}$, et al. Regional specificity of localized cortical lesions in West syndrome. Pediatr Neurol 2000; 23: 219-224.

23. Casetta I, Granieri E, Monetti VC, Gilli G, Tola MR, Paolino E, et al. Early predictors of intractability in childhood epilepsy: a communitybased case-control study in Copparo, Italy. Acta Neurol Scand 1999; 99: 329-333.

24. Berg AT, Levy SR, Novotny EJ, Shinnar S. Predictors of intractable epilepsy in childhood: a case-control study. Epilepsia 1996; 37: 2430.
25. Engel J Jr. Mesial temporal lobe epilepsy. In: Engel J Jr, Pedley TA (Editors), Epilepsy: A comprehensive textbook. Philadelphia: Lippincott-Raven; 1997. p 2417-2426.

26. Cendes F, Andermann F, Gloor P, Evans A, Jones-Gotman M, Watson $\mathrm{C}$, et al. MRI volumetric measurement of amygdala and hippocampus in temporal lobe epilepsy. Neurology 1993; 43: 719725.

27. French JA, Williamson PD, Thadani VM, Darcey TM, Mattson RH, Spencer SS, et al. Characteristics of medial temporal lobe epilepsy: I. Results of history and physical examination. Ann Neurol 1993; 34: 774-780.

28. Pitkanen A, Sutula TP. Is epilepsy a progressive disorder? Prospects for new therapeutic approaches in temporal-lobe epilepsy. Lancet Neurol 2002; 1: 173-181.

29. VanLandingham KE, Heinz ER, Cavazos JE, Lewis DV. Magnetic resonance imaging evidence of hippocampal injury after prolonged focal febrile convulsions. Ann Neurol 1998; 43: 413-426.

30. Mathern GW, Adelson PD, Cahan LD, Leite JP. Hippocampal neuron damage in human epilepsy: Meyer's hypothesis revisited. Prog Brain Res 2002; 135: 237-251.

31. Margerison $\mathrm{JH}$, Corsellis JA. Epilepsy and the temporal lobes. A clinical, electroencephalographic and neuropathological study of the brain in epilepsy, with particular reference to the temporal lobes. Brain 1966; 89: 499-530.

32. Briellmann RS, Newton MR, Wellard RM, Jackson GD. Hippocampal sclerosis following brief generalized seizures in adulthood. Neurology 2001; 57: 315-317.

33. Velasco TR, Wichert-Ana L, Walz R, Alexandre V Jr, Bianchin MM, Dalmagro CL, et al. Video-EEG monitoring in patients with normal MRI. Arq Neuropsiquiatr 2003; 61 (Suppl 1): 65-71.

34. Engel J Jr, Wiebe S, French J, Sperling M, Williamson P, Spencer D, et al. Practice parameter: temporal lobe and localized neocortical resections for epilepsy: report of the Quality Standards Subcommittee of the American Academy of Neurology, in association with the American Epilepsy Society and the American Association of Neurological Surgeons. Neurology 2003; 60: 538-547.

35. O'Donoghue M, Sander JW. Neurocysticercosis and epilepsy. Lancet 1993; 341: 568.

36. Cusmai R, Ricci S, Pinard JM, Plouin P, Fariello G, Dulac O. West syndrome due to perinatal insults. Epilepsia 1993; 34: 738-742.

37. Ohtahara S, Ohtsuka Y, Yamatogi Y, Oka E, Yoshinaga H, Sato M. Prenatal etiologies of West syndrome. Epilepsia 1993; 34: 716-722. 